\title{
The Role of Stress Granules and the Nonsense-mediated mRNA Decay Pathway in Antiviral Defence
}

\author{
Lara Contuad§, Silvio Steiner ${ }^{\mathrm{bcd}}$, Volker Thiel ${ }^{\star b c}$, and Oliver Mühlemann ${ }^{\star a}$
}

\begin{abstract}
Eukaryotic cells have evolved a number of survival tactics and quality control pathways that are able to counter intrinsic error-prone mechanisms and allow for maintenance of cellular homeostasis in the face of external stresses. This review will discuss the role of two cellular eukaryotic processes that are vital for maintenance of cellular homeostasis - 1) the nonsense-mediated mRNA decay (NMD) pathway and 2) the transient formation of stress granules (SG) - and explore the current literature on their roles in antiviral defence. Within the NCCR RNA \& Disease, the laboratories of Proffs. O. Mühlemann and Volker Thiel teamed up to unravel the roles of NMD and SGs, and their interconnections in defending cells from alphavirus and coronavirus infections.
\end{abstract}

Keywords: Innate immune response · Integrated stress response $\cdot$ Nonsense-mediated mRNA decay $\cdot$ RNA virus $\cdot$ Stress granules

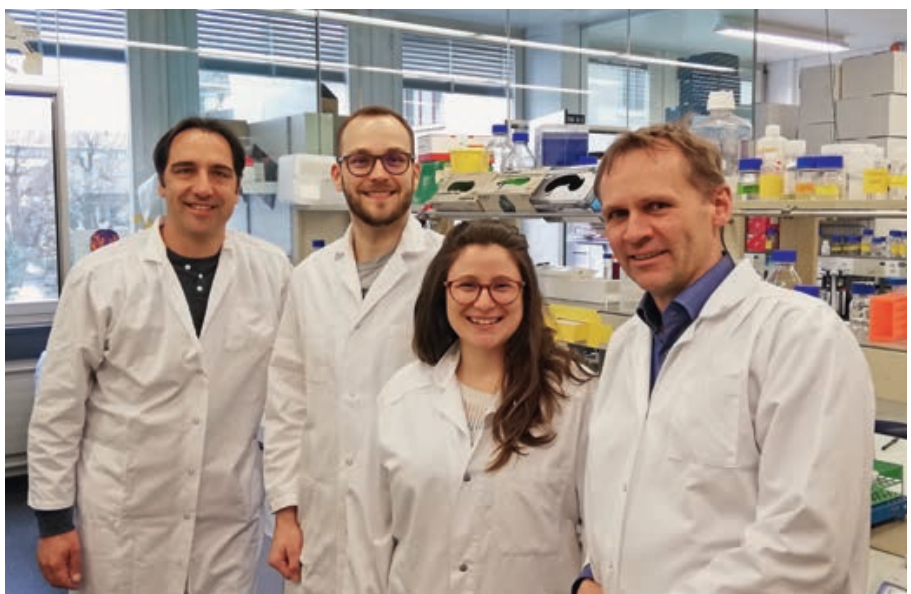

From left: Volker Thiel is professor of Veterinary Virology at the Vetsuisse Faculty, University of Bern, and Head of the division Virology of the federal Institute of Virology and Immunology (IVI). He did his $\mathrm{PhD}$ at the Institute of Virology, University of Würzburg, Germany, and led a research group as PI for 10 years at the Institute of Immunobiology at the Kantonal Hospital St.Gallen before he came to Bern. Silvio Steiner is a PhD student at the University of Bern, under the supervision of Volker Thiel. He did his undergraduate and Master studies in Molecular Life Sciences at the University of Bern. Lara Contu is a PhD student at the University of Bern, under the supervision of Oliver Mühlemann. She conducted her undergraduate and Masters studies in Biochemistry at Rhodes University in Grahamstown, South Africa. Oliver Mühlemann is professor of Biochemistry and the director of the NCCR RNA \& Disease. He did his PhD at the Karolinska Institute in Stockholm and at the Uppsala University, and a postdoc at the Brandeis University near Boston, before becoming a junior group leader at the University of Bern.

\section{The Role of NMD in Post-transcriptional Gene Regulation and mRNA Quality Control}

Nonsense-mediated mRNA decay (NMD) is best known for its role in mRNA quality control, where it rids the cytoplasm of transcripts with premature stop codons (PTCs) that could otherwise result in the translation of non-functional or potentially harmful, truncated proteins. ${ }^{[1,2]}$ Such PTCs could have arisen through nonsense or frameshift mutations in DNA, errors in transcription, or errors in splicing. ${ }^{[2]}$ PTCs could, however, also arise from alternative splicing events that produce multiple mRNA isoforms from a single gene. In many genes coding for splicing factors, these alternatively spliced PTC-containing transcripts are targeted by NMD, thereby linking the two processes in a mechanism referred to as alternative splicing-NMD (AS-NMD). ASNMD is physiologically relevant as the regulation of one splice variant versus another can modulate splicing, cell function/identity (reviewed in ref. [3]). AS-NMD therefore presents a distinct example of the role of NMD, not only in quality control, but also in post-transcriptional regulation of gene expression. It has become evident that this regulatory role of NMD extends beyond targeting of PTC-containing transcripts, with genome-wide data revealing that $\sim 10 \%$ of endogenous mRNAs in mammalian cells are targeted by NMD. ${ }^{[2,4,5]}$ Since the presence of a PTC does not account for all NMD-sensitive transcripts, the features/context that renders a mRNA a target of NMD remains an open question. Nevertheless, some features, including 3'UTR introns, long 3'UTRs, and upstream open reading frames (uORFs) have been shown to be enriched in endogenous NMD targets. ${ }^{[5]}$

It has long been established that NMD depends on translation, with a number of translation inhibitors (with different modes of action) all abrogating NMD. ${ }^{[6,7]}$ More specifically, evidence suggests that NMD is tightly coupled to translation termination; and the current NMD model posits that aberrant or kinetically slow translation termination is the NMD triggering event. ${ }^{[7-11]}$ It should 
be noted that poly(A)-binding protein (PABP) has been characterised to suppress NMD when in close proximity to the termination codon (TC). Accordingly, if PABP is distant from the TC (as is the case for mRNAs with long 3'UTRs), NMD can ensue. ${ }^{[12-14]}$ NMD activation and the ultimate degradation of target mRNAs depends on the dynamic assembly of a protein complex of which several evolutionarily conserved core NMD factors have been described. ${ }^{[7]}$ Interestingly, crosslinking immunoprecipitation (CLIP)-seq studies revealed that the central NMD factor, Up-frameshift 1 (UPF1), appears to bind RNA non-specifically, though significant enrichment of UPF1 bound to 3'UTRs was also observed.[15] A popular hypothesis postulates that when translation fails to terminate efficiently/correctly, it allows time for RNA-bound UPF1 to be activated by subsequent recruitment of downstream NMD factors. The NMD factors, UPF2 and/or UPF3 that are either free in the cytoplasm or positioned nearby on an exon junction complex (EJC), bind UPF1, thereby triggering a conformational change that allows UPF1 to be phosphorylated by the kinase SMG1. Phosphorylation of UPF1 allows for the subsequent recruitment of the NMDspecific degradation machinery (see Fig. 1 below). Namely, the SMG6 endonuclease which binds and degrades mRNA through its intrinsic ribonuclease activity, as well as the SMG5/SMG7 heterodimer which stimulates exonucleolytic mRNA degradation through recruitment of deadenylases and decapping enzymes. ${ }^{[7,16]}$ It should also be noted that the presence of an EJC downstream of a termination codon (as would be the case for mRNAs encoding an intron in the 3'UTR) has been reported to enhance NMD; although is not required in order for NMD to ensue. ${ }^{[2,17]}$

\section{Viral Genomes are Targeted by NMD}

Viruses depend on their hosts in order to proliferate. Positivesense single stranded RNA (+ssRNA) viruses are unique in that they depend on the host cell translation machinery immediately after entry into the cell so that their replicase proteins can be translated. Viruses have evolved to maximise their coding capacity while retaining relatively short genomes. ${ }^{[18]}$ For +ssRNA viruses, all proteins need to be encoded in a single strand with limited space. This means their genomes are often comprised of multiple ORFs, hence encompassing multiple TCs on a single RNA. During translation of the viral genomic RNA (gRNA), the internal TCs could be recognised as PTCs by the host NMD machinery. In addition, translation of the first ORF of a bi- or multi-cistronic +ssRNA genome could mimic translation of an mRNA with a long 3'UTR, which could also trigger NMD. ${ }^{[3,18]}$ Indeed, the genomes of several +ssRNA viruses have now been identified as NMD targets, ${ }^{[19-23]}$ prompting the view that NMD could play a role in innate antiviral defence. In 2014, Wernet and colleagues ${ }^{[19]}$ were able to increase titres of infectious virus-like particles (VLPs) of the alphavirus, Sindbis virus (SINV), produced in Drosophila, by co-expressing a dominant negative UPF1 mutant that abrogated NMD. Following that report, two back-to-back research articles in the same year reported the restriction of three additional +ssRNA viruses by NMD. $[20,21]$

Garcia and colleagues showed increased Potato Virus X (PVX) infection in NMD-deficient plants (N. benthamiana) compared to NMD-active plants. Additionally, northern blot analyses showed that two subgenomic (sg) RNAs of PVX (both with long 3'UTRs) had increased abundance in the NMD-deficient conditions compared to NMD-active conditions, whereas the 3' proximal sgRNA which did not have a long 3'UTR showed no changes in abundance. From this, and an additional experiment whereby they expressed a mutant of one of the sgRNAs that lacked the 3'UTR in NMD-deficient versus NMD-active conditions, the authors concluded that restriction of the sgRNAs by NMD was dependent on the presence of a long 3'UTR. ${ }^{[20]}$ Interestingly, the full length gRNA of PVX was only restricted by NMD in conditions where virus replication was impaired. It became evident that the replication of the full length genome masked the initial NMD-targeting event. ${ }^{[20]}$ Infection of $N$. benthamiana with Turnip Crinkle Virus (TCV), another +ssRNA virus that differs from PVX in that it lacks a 5'-cap and 3'-poly(A) tail, showed accumulation of full length gRNA as well as two sgRNAs (all with long 3'UTRs) in NMD-deficient compared to NMD-active conditions. ${ }^{[20]}$

Balistreri and colleagues found that depletion of the NMD factors, UPF1, SMG5 and SMG7 resulted in increased replication of the +ssRNA alphavirus, Semliki Forest Virus (SFV). This study was the first to identify additional NMD factors involved in viral restriction, thereby providing stronger evidence for the role of the NMD pathway itself in viral defence, as opposed to an NMD-independent role of UPF1. The increase in SFV replication in UPF1-depleted cells was strikingly pronounced when a replication-deficient SFV mutant was used.[21] This indicated that NMD was likely involved in hindering translation of early viral replicase proteins, possibly by degrading the incoming SFV genome. Indeed, DNA-branched fluorescence in situ hybridisation (FISH) studies performed 30 minutes post infection revealed that depletion of UPF1 increased the half-life of the SFV gRNA. ${ }^{\text {[21] The }}$ SFV genome is bi-cistronic, therefore translation of the first ORF mimics translation of an mRNA with a long 3'UTR. Surprisingly, an increase in viral replication in UPF1-depleted cells was also observed in SFV VLPs in which most of the 3'UTR was deleted ( $\Delta 3^{\prime}$ UTR SFV). ${ }^{[21]}$ It should be noted, however, that half-life measurements of the $\Delta 3^{\prime} \mathrm{UTR}$ SFV gRNA under these conditions were not performed.

Research into the role of NMD in antiviral defence is gaining traction, with studies in the current year adding both Mouse Hepatitis Virus (MHV) (a murine coronavirus) and Zika virus (ZIKV) to the growing list of +ssRNA viruses shown to be restricted by NMD. ${ }^{[22,23]}$ Virus replication and virus titres of MHV were significantly increased in cells depleted of the NMD factors, UPF1, UPF2, SMG5 and SMG6. ${ }^{[22]}$ Additionally, half-life measurements of MHV gRNA and an MHV sg-like RNA reporter revealed that NMD-depletion by UPF1 and UPF2 siRNAs increased viral RNA stability. This indicated that both the MHV gRNA and one of the sgRNAs (with a long 3'UTR) were both targeted by NMD. ${ }^{[22]}$ Similarly, depletion of UPF1 resulted in increased RNA levels and viral titres of the highly pathogenic ZIKV. ${ }^{[23]}$

The replication cycles of all the NMD-restricted viruses discussed above occur exclusively in the cytoplasm, with the genomes never being exposed to the host cell nuclear machinery. This is an interesting aspect, as it reflects the first clear example of splicing-independent and, by inference, EJC-independent NMD in mammalian cells - a model that was proposed a while ago ${ }^{[2,17]}$ but that has not yet been fully embraced by the NMD community.

\section{Inherent Viral Features Confer NMD Resistance}

For successful infection, viruses need to overcome the NMD machinery of their hosts. Turnip Mosaic Virus (TMV), a +ssRNA virus that encodes all proteins on a single ORF, was shown to be resistant to NMD. ${ }^{[20]}$ Viruses encoding single ORFs present no internal TCs and shorter 3'UTRs and it is therefore appealing to hypothesise that other viruses of this nature are inherently NMD resistant, although this did not hold true for ZIKV. ${ }^{[23]}$ Other examples of inherent viral features that confer NMD resistance have also been described. [24-27] For example, the retrovirus, Rous Sarcoma Virus (RSV), escapes NMD through a 400 nt RNA-stability element (RSE) encoded in its genome. ${ }^{[28,29]}$ The RSE was found to be a binding site for polypyrimidine tract binding protein 1 (PTBP1), which prevented UPF1 recruitment to otherwise NMD-sensitive reporter transcripts, thereby rendering them NMD-insensitive. ${ }^{[24]}$ It has been reported that NMD can be inhibited by inducing translational readthrough events. ${ }^{[26,30]}$ Many multicistronic viruses depend on readthrough-enhancing elements in their genomes in 
order to allow regulated translation of downstream ORFs. These inherent readthrough elements could therefore provide protection from NMD. Indeed, the RNA pseudoknot readthrough-enhancing element of the retrovirus, Moloney Murine Leukemia Virus (MoMLV), was able to rescue NMD-sensitive reporter transcripts from degradation by NMD. ${ }^{[25,26]}$ More recently, a ribosomal readthrough element identified from the +ssRNA virus, TCV; as well as a -1 frameshift-inducing element in the + ssRNA virus, Pea enation mosaic virus (PEMV2) were also shown to rescue NMDsensitive reporters when placed in a position/context that promoted readthrough. ${ }^{[27]}$

In addition, in 2018 May and colleagues identified an unstructured region (USR) in the 5' portion of the TCV 3'UTR that also conferred NMD resistance. ${ }^{[27]}$ The authors went on to show that the 3'UTRs of other carmoviruses, including Cardamine chlorotic fleck virus (CCFV), Carnation mottle virus (CarMV) and Calibrachoa mottle virus (CbMV) could also rescue NMDsensitive reporters, with TCV and CCFV 3'UTRs even maintaining NMD-resistance when placed downstream of a reporter with a longer 3'UTR. ${ }^{27]}$ Interestingly, introduction of mutations that introduced stable hairpins in the USR of TCV abrogated NMDresistance. ${ }^{[27]}$ The authors noted that endogenous transcripts with long 3'UTRs $>1400$ nt that escape NMD, have less structured regions $75 \mathrm{nt}$ downstream of the TC compared to endogenous NMD targets with long 3'UTRs. ${ }^{[27]}$ Since the context that renders a transcript an NMD target is still an open question in the field, this finding is significant as the presence of an unstructured region immediately downstream of a TC could explain why many endogenous mRNAs are protected from NMD despite having long 3'UTRs. ${ }^{[27]}$

\section{Viruses Actively Inhibit NMD}

In addition to specific features in viral genomes conferring resistance to NMD in cis, reports demonstrating global NMD inhibition by viral infection have also been described. [22,23,31,32] Human T-lymphotropic Virus Type 1 (HTLV-1) inhibits NMD through direct binding of its TAX protein to UPF1, thereby either preventing UPF1 association to RNA or disrupting UPF1 helicase activity. ${ }^{[32]} \mathrm{A}$ number of +ssRNA viruses that inhibit global NMD, including Hepatitis C Virus (HCV), MHV and ZIKV have also come to light. ${ }^{[22,23,31]}$ Infection with HCV was shown to increase levels of endogenous NMD reporters in hepatoma cells and it was postulated that binding of the $\mathrm{HCV}$ core protein to the EJC factor PYM could be the mechanism by which NMD was suppressed. ${ }^{[31]}$ In addition to its genome being a direct target of NMD, MHV suppressed NMD at later stages of infection. Expression of individual MHV proteins revealed that the nucleocapsid protein was responsible for the NMD-inhibitory phenotype. ${ }^{[22]}$ Since viruses are well known to inhibit global translation and NMD is dependent on translation, it could be hypothesised that viruses suppress NMD through suppression of translation. Wada and colleagues showed, however, that NMD targets accumulated 2 hours prior to observation of global translation inhibition. In addition, it was shown using luciferase reporters that expression of the MHV nucleocapsid did not reduce translation. ${ }^{[22]}$ Although no mechanistic insight was given, the study pointed towards an active role of NMD inhibition by the MHV nucleocapsid. ${ }^{22]}$ Infection of cells with the highly disease-relevant ZIKV also caused NMD inhibition. ${ }^{[23]}$ Using affinity purification coupled with mass spectrometry, the authors identified interactions of ZIKV capsid protein (from two different ZIKV strains) with many NMD factor components, including UPF1, UPF3B and a number of EJC components.[23] Strikingly, ZIKV capsid expression resulted in significant downregulation of UPF1 protein, while UPF1 transcript levels remained stable. Interestingly, the authors demonstrated that the nuclear UPF1 fraction was specifically downregulated and that proteasome-mediated deg- radation of UPF1 was occurring in the nucleus of ZIKV capsid expressing cells. ${ }^{[23]}$ Taken together, the authors suggested that ZIKV capsid specifically targets nuclear UPF1 for degradation by the proteasome. ${ }^{[23]}$ Since ZIKV infection during pregnancy is well established to cause microcephaly ${ }^{[33]}$ and genetic mutations that impair NMD have been described to be associated with microcephaly; ${ }^{[23,34-36]}$ the link between ZIKV infection and NMD suppression could shed light on the molecular mechanisms by which ZIKV causes disease. Taken together, the interplay between the host NMD pathway and viruses can be viewed as a tug of war between the virus establishing infection and the host cell contending to rid the cell of the viral stress with the ultimate goal of restoring homeostasis.

\section{The Integrated Stress Response and Stress Granules}

Like NMD, the integrated stress response (ISR) is a pathway in eukaryotic cells that is tightly linked to translational regulation. The ISR is tasked with the sensing of a multitude of extrinsic and intrinsic stresses and the subsequent reprogramming of translation in order to ultimately restore homeostasis. ${ }^{[37,38]}$ ISR responds to a variety of stressors such as hypoxia, amino acid deprivation, accumulation of unfolded proteins in the endoplasmic reticulum (ER), and viral infection. ${ }^{[37,39]}$ The hallmark feature of an activated ISR is the phosphorylation of the $\alpha$-subunit of the eukaryotic translation initiation factor $2(\mathrm{eIF} 2 \alpha) \cdot{ }^{[37,40]}$ The result of this phosphorylation causes a decrease in cap-dependent translation initiation on the majority of mRNAs, while simultaneously activating translation of select mRNAs. These stress-activated mRNAs encode for proteins tasked with restoring homeostasis and relieving the cell of the stress conditions. ${ }^{[38,41,42]}$ Prolonged stress, however, will lead to apoptosis if it overwhelms the cells adaptive capability. Dephosphorylation of eIF $2 \alpha$, in turn, acts as a stopping signal to the ISR response. ${ }^{[37,38]}$

In mammalian cells, eIF $2 \alpha$ can be phosphorylated by four distinct kinases, dependent on the kind of stress: Heme regulated eIF2 $\alpha$ kinase (HRI) is activated in response to oxidative stress and heme deprivation, ${ }^{[43]}$ while general control non-depressible-2 (GCN2) is activated upon amino acid deprivation and UV radiation. ${ }^{[44]}$ Protein kinase RNA-dependent (PKR) activates ISR in the presence of viral infections by sensing double-stranded (ds) RNA $^{[45]}$ and PKR-like ER kinase (PERK) is activated in response to misfolded proteins in the ER or by hypoxia. ${ }^{[46]}$ In its phosphorylated form, eIF $2 \alpha$ prevents the regeneration of the ternary complex (eIF2-GTP-tRNA ${ }_{\text {Met }}$ ) that is required for translation initiation. ${ }^{[38,47,48]}$ Instead, translation initiation is stopped after formation of the $48 \mathrm{~S}$ pre-initiation complex and the large ribosomal subunit cannot be recruited to start elongation. ${ }^{[49]}$ The result of that mechanism is an accumulation of stalled $48 \mathrm{~S}$ mRNPs, which are then concentrated into cytoplasmic foci called stress granules (SG, Fig. 1). ${ }^{[45]}$

SGs are dynamic structures that are in constant equilibrium with translating polysomes and thought to triage mRNAs during stress conditions. ${ }^{[47,50]} \mathrm{SGs}$ contain stalled mRNA molecules, $40 \mathrm{~S}$ ribosomal subunits, several translation initiation factors and RNA binding proteins, and they also appear to be hubs for a variety of signalling cascades. ${ }^{[48,50]}$ While there are other pathways that lead to SG formation, such as inhibition of the RNA helicase eIF4A, eIF $2 \alpha$ phosphorylation is the best studied pathway that leads to canonical stress granules. ${ }^{[51]}$ Increasing evidence suggests that SG formation is a multi-step process. ${ }^{[52]}$ First, a variety of RNA binding proteins (RBP), including T-cell restricted intracellular antigen 1 (TIA-1), TIA-1-related protein (TIAR), and RasGAP SH3-domain binding protein 1 (G3BP1), bind to non-translating 48S mRNPs and form dense, stable core aggregates. ${ }^{[48,52]}$ In a next step, intrinsically disordered regions (IDR), which are present in many RNA binding proteins (RBP) targeted to SGs, promote 


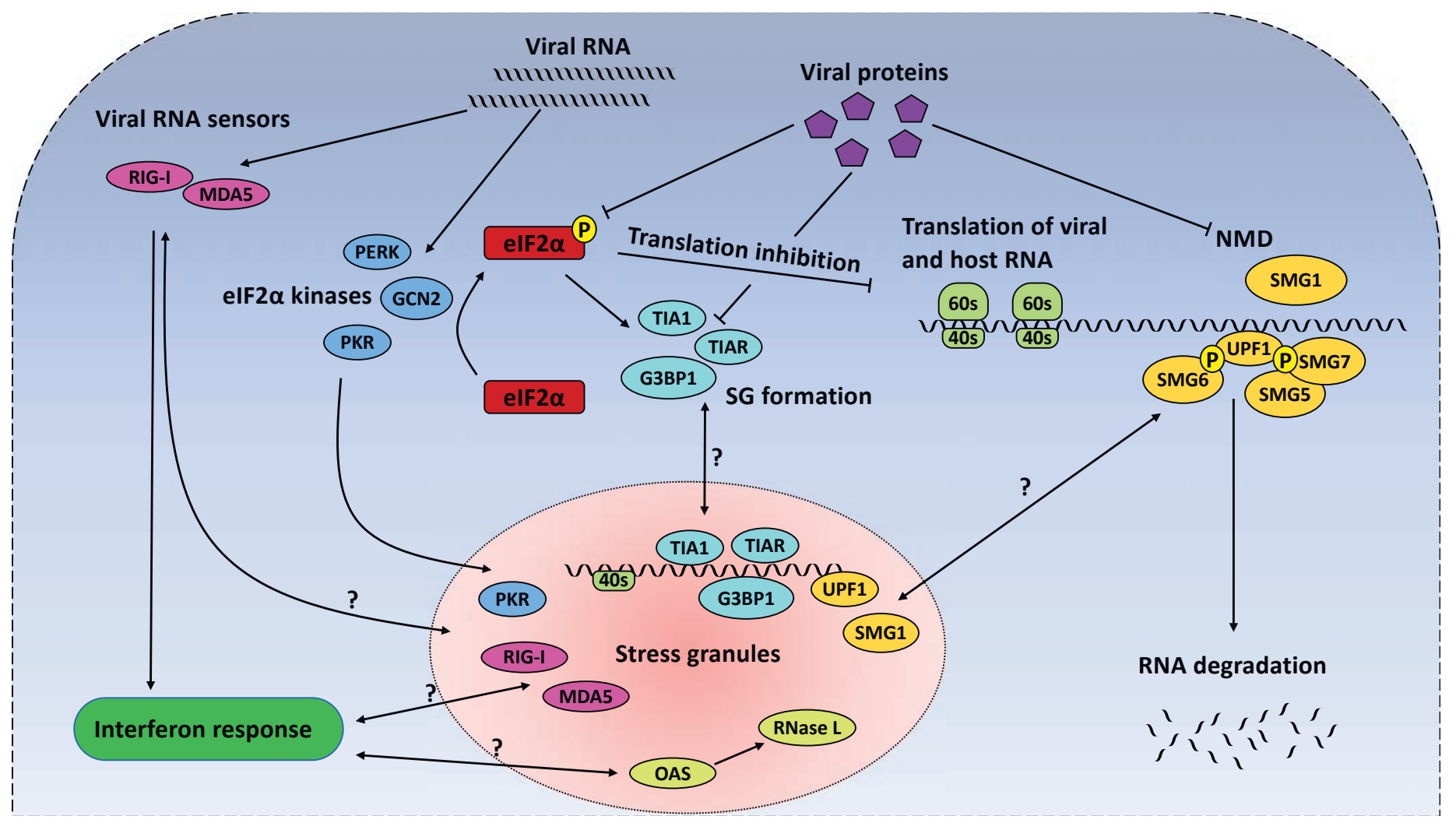

Fig. 1. The NMD pathway is dependent on translation and translation is stalled after SG formation. Critical host proteins that impact NMD, SG formation, and interferon responses are depicted. In order for a transcript to be degraded by NMD, UPF1 binds the transcript and is phosphorylated by the NMD factor kinase, SMG1. Phosphorylated UPF1 recruits the NMD degradation machinery. Namely, the SMG6 endonuclease and/or the SMG5/ SMG7 heterodimer, which stimulates degradation by recruitment of deadenylases and decapping enzymes. Various genomic and subgenomic RNAs of +SSRNA viruses have been described to be targeted by the NMD machinery, prompting the view that NMD could play a role in innate antiviral defence. Similarly, SGs have been proposed to play a role in innate antiviral defence mechanisms. Activation of elF2 $\alpha$ kinases can lead to SG formation through phosphorylation of elF2 $\alpha$. The presence of canonical SG markers (TIA1, TIAR, and G3BP1), components of the NMD pathway (UPF1, SMG1), dsRNA-specific PRRs (Rig-I, Mda5, PKR), and ISGs with antiviral activity (OAS, RNase L), suggests a link of these diverse cellular pathways in the context of antiviral host responses.

liquid-liquid phase separation to form a liquid-like layer around the stable cores. ${ }^{[48,52-54]}$ Held together by an interwoven network of weak interactions between proteins and RNA, these SGs fuse with one another and form huge compartments containing multiple cores. ${ }^{[48,52,55]}$

\section{Stress Granules and Viruses}

Since all viruses depend on the cellular translation machinery for their replication, it is not surprising that eukaryotic cells have developed means to restrict protein synthesis in infected cells. One of the key components for this antiviral translational reprogramming is the aforementioned eIF2 $\alpha$ kinase PKR. In the presence of dsRNA, which constitutes an important intermediary for RNA viruses, PKR dimerizes and phosphorylates itself. Besides dsRNA, other viral RNA motifs, such as 5'-triphosphate RNA. ${ }^{[45,48]}$ have been described to activate PKR as well. In addition, PKR is not the only eIF2 $\alpha$ kinase to play a role in antiviral defence, as both GCN2 ${ }^{[56]}$ and PERK ${ }^{[57]}$ have been implicated in activating the ISR in response to viruses. Once activated, PKR phosphorylates eIF $2 \alpha$ and triggers the ISR, which leads to the formation of SGs. SGs are intrinsically linked to the ISR and to translation, and many viruses have developed diverse countermeasures against these cellular defences. For example, Influenza A directly blocks activation of PKR and thus prevents the activation of the ISR. ${ }^{[58,59]}$ Other viruses, such as Poliovirus, specifically cleave essential SG proteins like G3BP1. ${ }^{[60,61]}$ An additional viral strategy is the sequestration of SG proteins, as shown for Semliki Forest Virus. [60,62] Yet other viruses, like MHV, just seem to tolerate SG formation and replicate normally in their presence. ${ }^{[58,63]}$ A strategy to circumvent the phospho-eIF2 $\alpha$-induced translation suppression is the use of internal ribosomal entry sites (IRES), which utilize cap-independent translation that is less sensitive to ISR activation. ${ }^{[48,64]}$ In cases where a virus loses its ability to control SG formation, for example because of a mutation in a viral protein, it was shown that viral propagation can be significantly reduced. ${ }^{[65,66]}$ Notably, several viruses have been reported to block SG formation downstream of translation inhibition even if they use IRES-based translation initiation. ${ }^{[48]}$ The finding that the presence of SGs is not required for ISR mediated translation inhibition suggests that SGs are more than a side effect of translation inhibition and may possess antiviral function.

\section{Stress Granules and Antiviral Responses}

The ISR is only one mode of action against viruses and the cell has additional lines of defence including the innate immune response. The innate immune response is activated upon recognition of pathogen-associated molecular patterns (PAMP), which in the case of viruses most often consist of their RNA or DNA. The sensors that bind these PAMPs are called pattern recognition receptors (PRR). In the case of virus recognition, PRRs include melanoma differentiation-associated gene 5 (MDA5), retinoic acid-inducible gene I (RIG-I) and laboratory of genetics and physiology 2 (LGP2), which are collectively called RIG-I-like receptors (RLR). If RLRs sense a viral infection in the cytoplasm, a signaling cascade is initiated that leads to the activation of transcription factors, interferon regulatory factor (IRF) 3, IRF7 and nuclear factor- $\kappa \mathrm{B}(\mathrm{NF} \kappa \mathrm{B})$. This, in turn leads to the production of the type 1 interferon (IFN) and other cytokines that initiate an extensive antiviral immune response via the expression of IFN stimulated genes (ISG). ${ }^{67-69]}$ 
In recent years, several reports have linked SGs to antiviral immune pathways. It was shown that RLRs but also PKR are recruited to SGs in the case of eIF2 $\alpha$ phosphorylation (Fig. 1). ${ }^{[70,71]}$ In the case of a mutant Influenza A virus (IAV), SGs were formed and colocalized with dsRNA sensors RIG-I, MDA5 and PKR, and SG formation was necessary for efficient type 1 IFN induction. ${ }^{\text {[70] }}$ Another report showed that a mutant Mengovirus induced SGs that recruited MDA5. ${ }^{[71]}$ In contrast to the report on IVA, however, SG formation was uncoupled to IFN expression in Mengovirus infection. ${ }^{[66,71]}$ In addition, several important cofactors to the RLR mediated IFN response, such as tripartite motif-containing protein 25 (TRIM25) ${ }^{[72]}$ or tumor necrosis factor receptor-associated factor 2 (TRAF2), ${ }^{[73]}$ were also found to localize to SGs. ${ }^{[48]}$ The finding of several viral RNA sensors and components of the corresponding signaling pathways recruited to SGs led to the hypothesis that SGs might function as signaling platforms for the innate immune system that may even be necessary for their activation. ${ }^{[48,70]}$ However, the extent to which IFN expression and PKR activation are connected is controversial. Some studies show that cells deficient for PKR have an impaired IFN response, while others found that PKR activation has no influence on IFN expression. ${ }^{[66]}$ Differences in cell types and viruses might account for this discrepancy, but more evidence is needed to solve this issue. Several ISG-encoded proteins were also found to colocalize with SGs in the case of virus infection. PKR is the ISG with the best documented link to SGs. While already expressed under normal conditions, PKR activity is enhanced upon IFN induction. Additionally, it has been shown that inactive PKR can be activated independently of dsRNA by interacting with the SG components G3BP1 and Caprin1. ${ }^{[74]}$ 2',5'-oligoadenylate synthetases (OAS) are another group of ISGs that were also found in SGs together with their effector protein RNase L. [70] Upon binding of dsRNA, the OAS proteins generate 2',5'-oligoadenylate, which activates RNase L. Once activated, RNase L degrades viral and cellular RNA. ${ }^{[69]}$ All these findings suggest that at least some parts of the antiviral immune response is linked to SGs. However, their specific role in that context is only partially understood and needs further study.

\section{A Connection between NMD and SGs in Defending Cells against Viruses?}

Interestingly, core components of the NMD pathway, UPF1 and SMG1, have been shown to localize to SGs induced by oxidative stress. ${ }^{[75]}$ It would be interesting to know whether NMD factors also localize to SGs that are induced by virus infections and whether a link exists between innate immune sensing, SGs, and NMD in the context of host cell responses to virus infections. Using SFV and coronavirus as models, the collaborative research project between the laboratories of Proffs. Mühlemann and Thiel is trying to answer exactly these questions with the goal of better understanding the molecular connections between SGs, NMD and their roles in protecting cells from virus infections.

\section{Acknowledgements}

The research of L.C. and S.S. is supported by the NCCR RNA \& Disease funded by the Swiss National Science Foundation (SNSF) and by the canton of Bern.

Received: March 1, 2019

[1] S. W. Peltz, A. H. Brown, A. Jacobson, Genes Dev. 1993, 7, 1737.

[2] L. Stalder, O. Mühlemann, Trends Cell Biol. 2008, 18, 315.

[3] S. Nasif, L. Contu, O. Mühlemann, Semin. Cell Dev. Biol. 2018, 75, 78.

[4] J. T. Mendell, N. A. Sharifi, J. L. Meyers, F. Martinez-Murillo, H. C. Dietz, Nat. Genet. 2004, 36, 1073

[5] M. Colombo, E. D. Karousis, J. Bourquin, R. Bruggmann, O. Mühlemann, RNA 2017, 23, 189 .
[6] M. Carter, J. Doskow, P. Morris, S. Li, R. P. Nhim, S. Sandstedt, M. F. Wilkinson, J. Biol. Chem. 1995, 270, 28995.

[7] E. D. Karousis, O. Mühlemann, Cold Spring Harb. Perspect. Biol. 2018, a032862.

[8] N. Amrani, R. Ganesan, S. Kervestin, D. A. Mangus, S. Ghosh, A. Jacobson, Nature 2004, 432, 112 .

[9] I. Peixeiro, Â. Inácio, C. Barbosa, A. L. Silva, S. A. Liebhaber, L. Romão, Nucleic Acids Res. 2012, 40, 1160.

[10] S. Kervestin, A. Jacobson, Nat. Rev. Mol. Cell Biol. 2012, 13, 700.

[11] F. He, A. Jacobson, Annu. Rev. Genet. 2015, 49, 339.

[12] P. V. Ivanov, N. H. Gehring, J. B. Kunz, M. W. Hentze, A. E. Kulozik, EMBO J. 2008, 27, 736 .

[13] A. B. Eberle, L. Stalder, H. Mathys, R. Z. Orozco, O. Mühlemann, PLoS Biol. 2008, 6, 849 .

[14] G. Singh, I. Rebbapragada, J. Lykke-Andersen, PLoS Biol. 2008, 6, 860

[15] D. Zünd, A. R. Gruber, M. Zavolan, O. Mühlemann, Nat. Struct. Mol. Biol. 2013, 20, 936.

[16] O. Mühlemann, J. Lykke-Andersen, RNA Biol. 2010, 7, 28.

[17] M. Bühler, S. Steiner, F. Mohn, A. Paillusson, O. Mühlemann, Nat. Struct. Mol. Biol. 2006, 13, 462.

[18] G. Balistreri, C. Bognanni, O. Mühlemann, Viruses 2017, 9, 1

[19] M. F. Wernet, M. Klovstad, T. R. Clandinin, Curr. Biol. 2014, 24, 107.

[20] D. Garcia, S. Garcia, O. Voinnet, Cell Host Microbe 2014, 16, 391.

[21] G. Balistreri, P. Horvath, C. Schweingruber, D. Zünd, G. McInerney, A. Merits, O. Mühlemann, C. Azzalin, A. Helenius, Cell Host Microbe 2014 $16,403$.

[22] M. Wada, K. G. Lokugamage, K. Nakagawa, K. Narayanan, S. Makino, Proc. Natl. Acad. Sci. 2018, 115, e10157.

[23] K. A. Fontaine, K. E. Leon, M. M. Khalid, S. Tomar, D. Jimenez-Morales, M. Dunlap, J. A. Kaye, P. S. Shah, S. Finkbeiner, N. J. Krogan, M. Ott, MBio 2018, 9,1 .

[24] Z. Ge, B. L. Quek, K. L. Beemon, J. R. Hogg, Elife 2016, 5, 1.

[25] X. Tang, Y. Zhu, S. L. Baker, M. W. Bowler, B. J. Chen, C. Chen, J. R. Hogg, S. P. Goff, H. Song, Nat. Commun. 2016, 7, 1

[26] S. L. Baker, J. R. Hogg, PLoS One 2017, 12, 1.

[27] J. P. May, X. Yuan, E. Sawicki, A. E. Simon, PLOS Pathog. 2018, 14, e1007459.

[28] J. E. Weil, K. L. Beemon, RNA 2006, 12, 102.

[29] J. B. Withers, K. Beemon, J. Cell Biochem. 2011, 112, 1.

[30] J. R. Hogg, S. P. Goff, Cell 2010, 143, 379.

[31] H. R. Ramage, G. R. Kumar, E. Verschueren, J. R. Johnson, J. Von Dollen, T. Johnson, B. Newton, P. Shah, J. Horner, N. J. Krogan, M. Ott, Mol. Cell 2015, 57, 329.

[32] F. Fiorini, J. Robin, J. Kanaan, M. Borowiak, V. Croquette, H. Le Hir, P. Jalinot, V. Mocquet, Nat. Commun. 2018, 9, 1.

[33] S. A. Rasmussen, D. J. Jamieson, M. A. Honein, L. R. Petersen, N. Engl. J. Med. 2016, 374, 1981.

[34] D. L. Silver, D. E. Watkins-Chow, K. C. Schreck, T. J. Pierfelice, D. M Larson, A. J. Burnetti, H. J. Liaw, K. Myung, C. A. Walsh, N. Gaiano, W. J. Pavan, Nat. Neurosci. 2010, 13, 551 .

[35] H. Mao, L.-J. Pilaz, J. J. McMahon, C. Golzio, D. Wu, L. Shi, N. Katsanis, D. L. Silver, J. Neurosci. 2015, 35, 7003.

[36] H. Mao, J. J. McMahon, Y. H. Tsai, Z. Wang, D. L. Silver, PLoS Genet. 2016, 12,1 .

[37] K. Pakos-Zebrucka, I. Koryga, K. Mnich, M. Ljujic, A. Samali, A. M. Gorman, EMBO Rep. 2016, 17, 1374

[38] G. D. Pavitt, Wiley Interdiscip. Rev. RNA 2018, 9, 1

[39] J. R. Buchan, R. Parker, Mol. Cell 2009, 36, 932.

[40] Y. Sekine, A. Zyryanova, A. Crespillo-Casado, P. M. Fischer, H. P. Harding, D. Ron, Science 2015, 348, 1027.

[41] S. K. Young, R. C. Wek, J. Biol. Chem. 2016, 291, 16927.

[42] A. G. Hinnebusch, I. P. Ivanov, N. Sonenberg, Science 2016, 352, 1413.

[43] A. P. Han, C. Yu, L. Lu, Y. Fujiwara, C. Browne, G. Chin, M. Fleming, P. Leboulch, S. H. Orkin, J. J. Chen, EMBO J. 2001, $20,6909$.

[44] T. E. Dever, L. Feng, R. C. Wek, M. A. Cigan, T. F. Donahue, A. G. Hinnebusch, Cell 1992, 68, 585.

[45] S. R. Nallagatla, J. Hwang, R. Toroney, X. Zheng, C. E. Cameron, P. C. Bevilacqua, Science 2007, 318, 1455

[46] H. P. Harding, Y. Zhang, D. Ron, Nature 1999, 397, 271.

[47] N. Kedersha, P. Anderson, Biochem. Soc. Trans. 2002, 30, 963.

[48] C. McCormick, D. A. Khaperskyy, Nat. Rev. Immunol. 2017, 17, 647.

[49] R. J. Jackson, C. U. T. Hellen, T. V. Pestova, Nat. Rev. Mol. Cell Biol. 2010, 11,113 .

[50] A. Aulas, M. M. Fay, S. M. Lyons, C. A. Achorn, N. Kedersha, P. Anderson, P. Ivanov, J. Cell Sci. 2017, 130, 927.

[51] L. C. Reineke, J. R. Neilson, Biochem. Pharmacol. 2018, in press.

[52] J. R. Wheeler, T. Matheny, S. Jain, R. Abrisch, R. Parker, Elife 2016, 5, 1.

[53] A. Molliex, J. Temirov, J. Lee, M. Coughlin, A. P. Kanagaraj, H. J. Kim, Cell 2015, 163, 123.

[54] Y. Lin, D. S. W. Protter, M. K. Rosen, R. Parker, Y. Lin, D. S. W. Protter, M. K. Rosen, R. Parker, Mol. Cell 2015, 60, 208. 
[55] N. Kedersha, P. Ivanov, P. Anderson, Trends Biochem. Sci. 2013, 38, 494.

[56] J. J. Berlanga, I. Ventoso, H. P. Harding, J. Deng, D. Ron, N. Sonenberg, L. Carrasco, C. De Haro, EMBO J. 2006, 25, 1730.

[57] G. Cheng, Z. Feng, B. He, J. Virol. 2005, 79, 1379.

[58] J. P. White, R. E. Lloyd, Trends Microbiol. 2012, 20, 175.

[59] D. A. Khaperskyy, T. F. Hatchette, C. McCormick, FASEB J. 2012, 26, 1629.

[60] N. Poblete-Durán, Y. Prades-Pérez, J. Vera-Otarola, R. Soto-Rifo, F. Valiente-Echeverría, Viruses 2016, 8, 1.

[61] J. P. White, A. M. Cardenas, W. E. Marissen, R. E. Lloyd, Cell Host Microbe 2007, 2, 295.

[62] M. D. Panas, M. Varjak, A. Lulla, K. Er Eng, A. Merits, G. B. Karlsson Hedestam, G. M. McInerney, Mol. Biol. Cell 2012, 23, 4701.

[63] M. Raaben, M. J. A. Groot Koerkamp, P. J. M. Rottier, C. A. M. de Haan, Cell. Microbiol. 2007, 9, 2218.

[64] E. Jan, I. Mohr, D. Walsh, Annu. Rev. Virol. 2016, 3, 283

[65] H. Katoh, T. Okamoto, T. Fukuhara, H. Kambara, E. Morita, Y. Mori, W. Kamitani, Y. Matsuura, J. Virol. 2013, 87, 489.

[66] K. Onomoto, M. Yoneyama, G. Fung, H. Kato, T. Fujita, Trends Immunol. 2014, 35, 420 .
[67] S. Reikine, J. B. Nguyen, Y. Modis, Front. Immunol. 2014, 5, 1

[68] H. J. Ramos, M. Gale, Curr. Opin. Virol. 2011, 1, 167.

[69] W. M. Schneider, M. D. Chevillotte, C. M. Rice, Annu. Rev. Immunol. 2014, 32,513 .

[70] K. Onomoto, M. Jogi, J. S. Yoo, R. Narita, S. Morimoto, A. Takemura, S. Sambhara, A. Kawaguchi, S. Osari, K. Nagata, T. Amtsumiya, H. Namiki, M. Yoneyama, T. Fujita, PLoS One 2012, 7, e43031.

[71] M. A. Langereis, Q. Feng, F. J. van Kuppeveld, J. Virol. 2013, 87, 6314.

[72] M. . Sànchez-Aparicio, J. Ayllon, A. Leo-Macias, T. Wolff, A. Garcia-Sastre, J. Virol. 2017, 91, 1

[73] W. J. Kim, S. H. Back, V. Kim, I. Ryu, S. K. Jang, Mol. Cell. Biol. 2005, 25 , 2450.

[74] L. C. Reineke, N. Kedersha, M. A. Langereis, F. J. M. van Kuppeveld, R. E. Lloyd, MBio 2015, 6, e02486.

[75] J. A. L. Brown, T. L. Roberts, R. Richards, R. Woods, G. Birrell, Y. C. Lim S. Ohno, A. Yamashita, R. T. Abraham, N. Gueven, M. F. Lavin, Mol. Cell. Biol. 2011, 31, 4417. 